Cahiers d'études italiennes

\title{
Alessandro Piperno : du judaïsme à la judéité. À la recherche du juif perdu
}

Sophie Nezri-Dufour

\section{OpenEdition}

\section{Journals}

Édition électronique

URL : http://journals.openedition.org/cei/198

DOI : $10.4000 /$ cei. 198

ISSN : 2260-779X

Éditeur

UGA Éditions/Université Grenoble Alpes

\section{Édition imprimée}

Date de publication : 15 juillet 2009

Pagination : 127-132

ISBN : $978-2-84310-145-8$

ISSN : 1770-9571

\section{Référence électronique}

Sophie Nezri-Dufour, «Alessandro Piperno : du judaïsme à la judéité. À la recherche du juif perdu », Cahiers d'études italiennes [En ligne], 9 | 2009, mis en ligne le 15 janvier 2011, consulté le 27 mars 2021. URL : http://journals.openedition.org/cei/198 ; DOI : https://doi.org/10.4000/cei.198 


\title{
ALESSANDRO PIPERNO : DU JUDAÏSME À LA JUDÉITÉ. À LA RECHERCHE DU JUIF PERDU
}

\author{
Sophie Nezri-Dufour \\ Université Aix-Marseille I
}

L'un des derniers événements littéraires italiens est la sortie du roman très controversé d'Alessandro Piperno, auteur du best-seller Con le peggiori intenzioni, publié chez Mondadori en $2005^{1}$. Dans ce roman iconoclaste, sarcastique, impertinent, Piperno a élaboré un personnage haut en couleur : protagoniste-narrateur, Daniel Sonnino est un jeune juif de la bourgeoisie romaine qui décortique et analyse avec délectation et masochisme sa propre judéité. Masochisme en effet car peut-on parler de judéité dans le cas de ce jeune Woody Allen romain ? Au sens strict du terme, et selon la loi juive, il n'est pas juif puisque sa mère ne l'est pas. C'est d'ailleurs là que réside le drame de tout le roman et que se situent les ressorts de la tragi-comédie que Piperno met alors savamment en place et dans laquelle, sans tomber dans le piège qui constituerait à identifier totalement Sonnino à Piperno, il est évident que des similitudes frappantes existent entre les deux individus : la mère de Piperno elle-même n’est pas juive, et Piperno n'est donc pas considéré comme tel aux yeux des autorités rabbiniques.

Sous un certain angle, on pourrait même considérer son roman comme une sorte de lettre ouverte aux autorités religieuses, écrite par un individu qui revendique sa judéité, marginale certes, mais profondément ancrée en lui.

Si Daniel Sonnino, l'alter ego de Piperno, se sent en effet profondément juif, c'est parce qu'il se sent lié à un judaïsme qu'il considère non pas tant comme une religion que comme une culture à laquelle il se sent indéniablement attaché, tout en la jugeant avec une profonde ironie et un regard qui se veut désacralisateur, démythificateur. Piperno sait bien que

I. A. Piperno, Con le peggiori intenzioni, Milan, Mondadori, 2005, 304 p. 
le judaïsme n'est pas qu'une réalité religieuse, mais également historique et culturelle : il s'engouffre alors avec fierté et insolence dans cette brèche salvatrice qui va lui ouvrir les portes du Paradis. Et pourtant, quel paradis! Un paradis juif digne des plus belles comédies de Woody Allen, ou, plus sérieusement, des plus frappantes contradictions que connaît le juif depuis qu'il vit en Diaspora.

Avec Piperno, le lecteur se retrouve en effet dans une véritable reconstruction identitaire très personnelle où le judaïsme offre un visage particulièrement surréaliste. Car chez Piperno s'impose avec force et paroxysme un phénomène qui existe depuis la sortie des ghettos, à savoir une dissociation exacerbée des diverses composantes du judaïsme, dans laquelle certaines d'entre elles, culturelle ou politique, priment largement sur la dimension religieuse, voire s'affirment contre elle. Ainsi, comme un certain nombre de juifs, le juif pipernien, bien que ne répondant à aucun des critères religieux ordinairement exigées, et ne pratiquant nullement le judaïsme, ne s'en réclame pas moins vigoureusement juif ${ }^{2}$.

Comme nous allons le voir en effet dans le roman de Piperno, le lecteur assiste à un glissement très net entre judaïsme et judéité, une judéité en outre schizophrénique, c'est-à-dire à la fois très iconoclaste et très soucieuse d'intégration et de reconnaissance. L'obsession du protagoniste est en effet d'être considéré comme juif, envers et contre tout, même s'il ne l'est pas. Et de nombreuses scènes du roman tournent autour de ce drame que Piperno nous présente toujours sous forme désopilante et sarcastique, refusant tout pathos, un pathos qui serait étranger à sa position de râleur patenté. Ainsi, lorsque le grand-père juif du protagoniste, décédé, est mis en terre, la cérémonie religieuse ne peut avoir lieu car, malgré la présence de plusieurs dizaines de personnes, il devient impossible de réunir un minian, c'est-à-dire le quorum nécessaire composé de dix juifs pour réciter la prière du kaddish :

Solo alle soglie del kaddish ci si accorge che tra i convenuti ci sono soltanto nove ebrei adulti. Ne manca uno per comporre il minian, numero minimo di dieci maschi adulti per poter eseguire le funzioni. Mio fratello e io siamo esclusi, non essendo ebrei. Mio padre e mio zio sono costernati, mentre il rabbino Perugia [...] torna a fare la conta nella speranza di trovare qualcuno che abbia i requisiti. Ma lo spettacolo è [...] all'occhio d'un vero ebreo, avvilente: una banda di marrani, convertiti, sangue misto a iosa, atei d'estrazione marxista [...]. (Piperno, p. 43)

2. J.-C. Attias et E. Benbassa, Dictionnaire de la civilisation juive, Paris, Larousse-Bordas, coll. « Les référents ", 1997, p. I27-I28. 
La farse continue lorsque le jeune protagoniste, Daniel, qui n'est pourtant pas juif selon la loi mosaïque, offre sa participation au minian : " E' ora di farsi avanti! mi intima imperiosamente una voce interna. "Ci sono io!" azzardo, e lo dico con un insorgente orgoglio. " Avec quel résultat? Son père se plie littéralement en deux, victime d'un terrible fou-rire (Piperno, p. 45). À travers cette scène qui se veut comique, et qui l'est en partie, Piperno souligne l'existence en Italie et en Europe d'une véritable réalité sociologique, vécue parfois douloureusement par de très nombreux enfants de père juif et de mère chrétienne, non intégrés dans la communauté ancestrale, et pourtant très attachés à cette culture qui fait partie de leur identité :

Mio padre è stato chiaro. Tu non sei ebreo! Non è la prima volta che se ne esce così [...] che mi insulta in questo modo [...]. Un'esecrabile corruzione nel DNA stava sfrattando un povero bambino di dieci anni dal suo spicchio di eternità! [...] devo ingoiare questo rospo assurdo: semplice cruda verità storica: Tu non sei ebreo! [...] Questa è semplicemente la tua condanna: essere ebreo per i gentili e gentile per gli ebrei! Né c'è da stupirsi che qualcuno, benché ancora adolescente, desideri ardentemente essere ebreo. Non c'è da sbalordirsi che un bambino voglia essere come suo padre. Un ebreo come tanti altri (p. 46-47),

ajoute le narrateur ironiquement. Ironiquement en effet car l'Histoire a bien changé. Alors qu'il était plutôt préférable à une époque ne pas être ou tout au moins ne pas se révéler juif, il est aujourd'hui très à la mode, en Italie en tout cas, de se déclarer comme tel : "Perché oggi è uno spasso essere ebrei. Compianto, accudito, esaltato: ecco la troika verbale per definire la condizione dell'ebreo contemporaneo" (p. 48), écrit Piperno, avec beaucoup de discernement et de sarcasme en même temps. Car il sait qu'il traite un thème à la mode, qui a mis du temps à s'imposer dans la Péninsule, mais qui, aujourd'hui, fait recette. Il l'exploite donc, sans s'en cacher, mais dans une perspective humoristique et iconoclaste systématique : "C'è gente che, contro ogni logica", écrit-il,

fa ricerche per accreditare la propria discendenza, non dall'ennesimo conto o marchese imparruccato, ma da un pio israelita cinquecentesco. Un tipetto alla Montaigne, tutta casa e famiglia. Da non crederci. Un ebreo nell'albero genealogico: il grande sogno distintivo del ventunesimo secolo. L'araldica del Nuovo Millennio. La griffe capace di renderti dolentemente salottiero e civilmente provocatorio (p. 48).

Ce qui n'empêche pas au narrateur, à plusieurs reprises, d'affirmer sa fierté d'appartenir à une famille juive, malgré le regard critique qu'il jète sur ceux qui exploitent ce filon. Il n'y a qu'à lire certains passages comme celui de la rencontre entre la famille juive paternelle et la famille chrétienne de la mère de Daniel : l'ambiance est évidemment électrique et les préjugés antisémites fusent. Ainsi, le grand-père chrétien d'expliquer à la belle-famille : 
Avremmo preferito [...] che Fiamma sposasse un ragazzo italiano!

Italiano? In che senso?

Perché, Luca non sarebbe italiano?

Beh, insomma, ha capito cosa intendevo... D'altronde mi ha detto mia figlia che Luca non ha fatto il militare... e neanche lei, se mi permette [...].

È vero; né io né mio figlio abbiamo fatto il militare. Ma per motivi affatto diversi da quelli che lei evidentement immagina. E non certo perché non siamo italiani... Anche se potrà stupirla, noi siamo italiani tanto quanto lei!

E allora?

A me lo hanno impedito le leggi razziali. Ma credo di aver servito il mio Paese. Io sono stato partigiano. (p. III)

Le drame de Daniel est ainsi de n'être à sa place nulle part. Non intégré dans la communauté juive, il est rejeté par les non-juifs qui eux le considèrent beaucoup trop juif. Son grand-père maternel, donc chrétien, a lui-même beaucoup de mal à le considérer comme faisant partie de sa descendance :

[...] vedeva in me, forse per la mia somiglianza con mio padre, forse per i tratti ebraici che portavo scolpiti sul mio volto, il figlio della colpa. L'incarnazione stessa dello sbaglio di mia madre [...] d'un tratto mi guardava e diceva a mia madre: "Daniel è furbo, stai attenta, è furbo, già ti sei fatta infinocchiare una volta... Hai visto che naso? [...] Lo stesso naso di suo padre e di suo nonno...” E non la smetteva di ridere di questa constatazione fisionomica che gli sembrava così ineccepibilmente rivelatrice.

Non-juif chez les juifs, juif chez les non-juifs, le pauvre Daniel Sonnino vit une contradiction identitaire tragi-comique qu'il tente d'évacuer à travers le rire : "Credo di essere il primo ebreo della storia dell'umanità ", explique-t-il, " ad aver subito discriminazioni dal proprio stesso nonno. Il primo ebreo della storia con un nonno antisemita" (p. I07-I08). Mais Piperno a fait son choix, qui est de se rattacher malgré le regard iconoclaste qu'il lui porte, à un univers juif dans lequel il trouve un territoire culturel historique et identitaire qui lui convient, au point de s'opposer aux penchants assimilationnistes de certains juifs. Ne regrette-il pas en effet l'attitude de la mère de David, fille de déportés, qui pour oublier le traumatisme de la Shoah, tente d'occulter son appartenance au judaïsme, alors qu'elle est pourtant mariée à un juif :

Karen si è imposta due missioni tra loro collegate: da un lato degiudaizzare Amos [il marito], dall'altro creare dal nulla un rispettabile pedigree per Dav [...] Karen sta esagerando: che faccia pure di suo figlio un gentile, che lo mandi a scuola dai preti, che gli faccia osservare il Natale, la Pasqua, il mercoledì delle Ceneri, che gli faccia frequentare tutte quelle insipide biondine, che riempia la casa di tutti quei chiusi snob; ma perché la sua tracotanza deve estendersi al punto di impedire a lui, Amos 
Ruben, di vivere a suo modo, così come gli hanno insegnato? [...] Lui è ebreo. E' fuggito da un Paese e da un'epoca nei quali tale pecularietà era considerata un problema. Proprio per questo ora non permetterà a nessuno [...] di impedirgli di vivere come tale. Sente l'esigenza di onorare le feste comandate e di sostenere economicamente i suoi parenti sparsi per l'Europa. (p. I7I)

Les pires intentions de Piperno, pour reprendre le titre de son roman, sont donc d'apporter un nouveau souffle à une réflexion sur l'identité juive dépourvue de tabous et de sous-entendus. De proposer un roman où l'individu juif, au-delà des stéréotypes positifs ou négatifs qui l'entourent, soit considéré comme un italien à part entière, avec ses limites, ses névroses et ses particularités. Piperno veut intégrer le juif dans la réalité italienne, et dans la toute nouvelle littérature italienne, en en faisant un citoyen de la Péninsule comme les autres, avec son histoire particulière certes, mais dans une perspective qui le sorte de cette mythification liée à la Shoah qui faisait du juif un personnage toujours victime, sympathique, mais difficilement acteur de son existence. Piperno offre en effet une réflexion sur la judéité sans fausse pudeur, volontairement iconoclaste, désacralisatrice, sans crainte de froisser les susceptibilités. Car les juifs, dans son roman, sont particulièrement désinvoltes et privés de toute morale. Prétentieux, érotomanes, aimant l'argent et la bonne chère, ils sont loin d'être les représentants modérés et vertueux d'une éthique juive que l'on peut retrouver dans certaines pages d'un Primo Levi ou d'un Bassani.

Comme nous l'avons vu, la réalité juive est volontairement démythifiée par Piperno, sans doute pour qu'un hybride tel que lui y fasse plus facilement sa place. Car le but de Piperno est non seulement d'être reconnu comme un membre de la communauté juive, mais aussi comme un nouvel écrivain juif italien. N'évoque-t-il pas le profond sentiment de se sentir "parte di qualcosa di più grande di [lui], una sorta di ultimo discendente di questa famiglia-tribù, di questi nani, rancidi bizantini alla mercé della loro ultima stagione, questi gagà semiebrei scampati allo sterminio». Il se définit en effet, à leur égard, "un bilioso curatore testamentario [...] uno di quegli etilisti pellerossa che bivaccano nelle sempre più anguste riserve americane nel culto e nel vagheggiamento di tempi che non possono più tornare " (p. 42-43).

Cette référence à l'Amérique n'est pas sans nous rappeler également la présence sous-jacente des écrivains juifs américains qui ont été, selon Piperno lui-même, " $i$ numi tutelari " de son roman. Comment en effet ne pas reconnaître l'influence de Philip Roth, à travers son protagoniste fétichiste des dessous féminins, nourrissant des rapports problématiques avec son identité juive et la communauté dont il fait partie, mais qu'il 
tourne en ridicule. Sa veine ironique, et auto-ironique, caustique, révèle une empreinte judéo-américaine évidente : avec Piperno, la provocation, le sarcasme et l'auto-flagellation font partie d'une démarche et d'un style destinés à mettre à nu et en spectacle, sans aucun respect mais finalement avec beaucoup de tendresse cachée, un univers juif qu'il offre à la curiosité du lecteur peu habitué, en Italie, à voir chez les écrivains juifs, une telle désinvolture et, finalement, un tel optimisme.

La réélaboration identitaire du juif Piperno se fait donc à plusieurs niveaux : bien que non-juif stricto sensu, il recrée une réalité et un univers juifs dans lesquels il s'inclut de plein droit et dont il montre connaitre les ressorts et les enjeux à travers des thèmes tels que la Shoah, l'assimilation, Israël, l'antisémitisme ; au niveau littéraire aussi, en offrant l'exemple d'un voyage dans l'univers juif à l'égard duquel il porte un regard certes iconoclaste, mais finalement profondément empathique. 